\title{
Copenhagen Adduction Exercise to Increase Eccentric Strength: A Systematic Review and Meta-Analysis
}

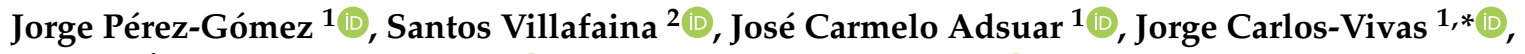 \\ Miguel Ángel Garcia-Gordillo ${ }^{3}$ (D) and Daniel Collado-Mateo ${ }^{4}$ (1) \\ 1 Health, Economy, Motricity and Education (HEME) Research Group, Faculty of Sport Sciences, \\ University of Extremadura, 10003 Cáceres, Spain; jorgepg100@gmail.com (J.P.-G.); \\ carmelo.adsuar@gmail.com (J.C.A.) \\ 2 Physical Activity and Quality of Life Research Group (AFYCAV), Faculty of Sport Sciences, \\ University of Extremadura, 10003 Cáceres, Spain; svillafaina@unex.es \\ 3 Facultad de Administración y Negocios, Universidad Autónoma de Chile, Sede Talca 3467987, Chile; \\ miguelgarciagordillo@gmail.com \\ 4 Centre for Sport Studies, Rey Juan Carlos University, Fuenlabrada, 28943 Madrid, Spain; \\ danicolladom@gmail.com \\ * Correspondence: jorge.carlosvivas@gmail.com
}

Received: 2 April 2020; Accepted: 17 April 2020; Published: 21 April 2020

check for updates

\begin{abstract}
Background: The purpose of this study was to systematically review the scientific literature about the benefits of using the Copenhagen Adductor (CA) exercise to improve eccentric hip adduction strength (EHAD), as well as to provide directions for training. Methods: A systematic search was conducted in three electronic databases following Preferred Reporting Items for Systematic Reviews and Meta-Analyses (PRISMA) guidelines. The inclusion criteria were: (a) EHAD as outcome variable, with means and standard deviations before and after the intervention, (b) the study was a randomized controlled trial using the CA in the program intervention, (c) the article was written in English. The quality of evidence was evaluated according to the Grading of Recommendations, Assessment, Development and Evaluation (GRADE) guidelines. A mini meta-analysis was performed to determine the overall effect. Results: Three studies were selected and included in this systematic review and mini meta-analysis. All articles reported significant enhancement in EHAD in football (soccer) players. The overall effect for the EHAD test was significant in favor to CA group (mean difference $=0.61$; $95 \%$ CI from 0.20 to 1.01; $p=0.003$ ) with large heterogeneity. Conclusions: CA exercise performed 2-3 times for 8 weeks is useful for improving EHAD in football players.
\end{abstract}

Keywords: groin injuries; hip adduction strength; injury prevention; football; soccer

\section{Introduction}

Muscle injuries are common in many sports, such as basketball [1], rugby [2], football [3], tennis [4], track and field [5]. Muscle injury can represent more than $40 \%$ of total injuries in football [3,6], and track and field [5]. Ice hockey and football are susceptible to many adductor muscle strains [7]. In this regard, the adductor injury is the second most common muscle injury among football players [3] and is associated with lost competition time [8]. Therefore, intervention programs aimed to reduce the incidence of adductor muscle injuries are recommended.

The low level of adductor muscle strength has been linked to the possibility of suffering an injury for instance in ice hockey, where the muscle strength ratio between adduction and abduction has been reported as a risk factor [9]. Tyler et al. also found that adduction strength was lower (18\%), during the preseason in players who sustained groin strains compared with uninjured players [9]. 
Furthermore, large adductor eccentric contraction demands in sports training and competition seem to be one of the reasons to have a high prevalence of adductor injuries [10]. Thus, many different exercises have been included in preventive programs [11,12] focused on improving adductor strength, in order to avoid injuries and limit the amount of losing time in competition [13] including, among others, adduction partner, hip adduction with an elastic band, isometric adduction, sliding hip abduction/adduction exercise, side-lying adduction and the Copenhagen adduction (CA) exercise.

The CA exercise is a dynamic high-intensity exercise that does not require equipment and can be easily performed on-field and at any training facility [14]. It is an eccentric partner exercise performed to outer range of motion, the player who is exercising is in a side-lying position using the forearm as support on the floor, the other arm is placed along the body, the upper leg is held by the partner, approximately at the height of his hip, with one hand on the ankle and the another one on the knee. Then, the player must raise the body from the floor, around $3 \mathrm{~s}$ concentric hip adduction, until the body is in a straight line, so the feet are touching each other. Then the body is lowered halfway to the ground, around $3 \mathrm{~s}$ eccentric adduction, until touching the floor without using it for support [12].

Although the high prevalence of adductor injuries and the potential benefits of the CA exercise, to our knowledge there are no meta-analyses aimed to analyze the effect of CA on eccentric hip adduction strength (EHAD). Therefore, the purpose of this study was to systematically review the scientific literature about the effect of CA on EHAD and to provide practical recommendations for training. Besides, a mini meta-analysis was carried out to determine the effect sizes of CA on EHAD.

\section{Materials and Methods}

The review was conducted following the statements of the Preferred Reporting Items for Systematic Reviews and Meta-Analyses guidelines (PRISMA) [15].

\subsection{Literature Search}

To find the studies reported in this review, several electronic databases were screened: PubMed (Medline), Web of Science (which includes other databases such as Current Contents Connect, Derwent Innovations Index, Korean Journal Database, Russian Science Citation Index and SCIELO citation index) and Google Scholar. The searches were limited to studies published up to and including 2 March 2020. The search terms were: (a) the type of exercise ("Copenhagen adduction") and (b) the word ("randomized"). The search was conducted using the type of exercise and the word separated by the Boolean operator "AND". Figure 1 shows the flow chart for selection of studies according to PRISMA statements.

\subsection{Study Selection}

The inclusion criteria were: (a) EHAD as outcome measure, reporting means and standard deviations (SD) before and after the intervention, (b) the study was a randomized controlled trial (RCT) using the CA in the program intervention, (c) the article was written in English. Two independent authors selected the potentially eligible articles from the databases. There were no disagreements.

\subsection{Quality of the Evidence}

The quality of the evidence was categorized using the Grading of Recommendations, Assessment, Development and Evaluation (GRADE) approach [16]. The evidence of the effects on EHAD was initially classified as "high quality" due to all the selected articles were RCT, but the evidence was downgraded once because of the small sample size and once because of the potential risk of bias. Therefore, the final quality of the evidence was low. 


\subsection{Data Collection}

Two authors independently extracted data from the studies. The information included: participants, interventions, comparisons, outcomes, and study design (PICOS), following the recommendations from the PRISMA statement. Table 1 shows age, sex, sample size and condition of the participants. Table 2 presents intervention and the comparison groups, including the type of training intervention, duration of the study, sessions per week, number of sets, repetitions, weekly volume and when the intervention was performed during the season. Figure 2 displays results for the different outcomes. The study design was not included in any table because all studies were RCT.

\subsection{Statistical Analysis}

The primary outcome of this mini meta-analysis was the EHAD test. Figure 2 displays the results of each study on this variable. All analyses were performed using the Review Manager Software (RevMan, 5.3, Cochrane Collaboration, Oxford, UK). The analysis method used was inverse variance and random effects due to the heterogeneity of articles [17]. The mean difference was used for EHAD. The results obtained where represented with a confidence interval (CI) of $95 \%$. Heterogeneity has calculating by the $\mathrm{I}^{2}$ statistic model and for the overall effect, Z-test was used $[18,19]$. Although there is no consensus about the definition of "mild", "moderate" or "severe" heterogeneity, Higgins and Thompson suggested that $\mathrm{H}$ values exceeding 1.5 might indicate severe heterogeneity, while values below 1.2 might represent low heterogeneity. Similarly, they proposed $\mathrm{I}^{2}$ of $56 \%$ as cutoff for large heterogeneity and $31 \%$ for low heterogeneity [20].

\section{Results}

\subsection{Study Selection}

PRISMA flow diagram is showed in Figure 1. A total of 126 records were identified, eight of which were removed because they were duplicates. Of the remaining 118 articles, 97 were excluded because they were not related to the topic, five studies were not written in English and three were congress abstracts or books. After reading the remaining 13 articles, another 10 studies were excluded because they did not include EHAD as outcome measure, not report means and SD before and after the intervention or they were no RCT that applied the CA in the training program. Finally, three studies were included in the meta-analysis. 


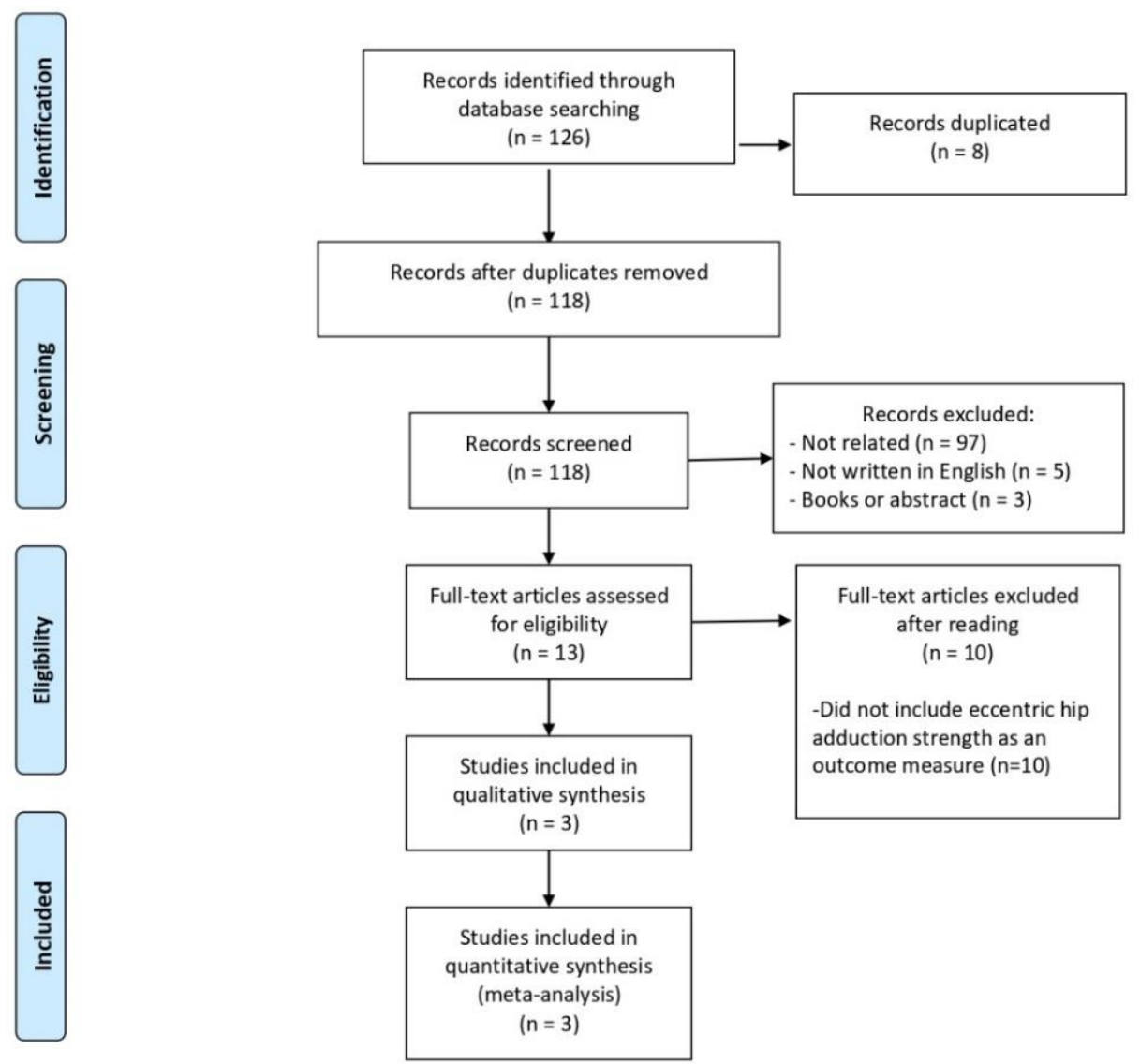

Figure 1. Flow chart for selection of studies according to PRISMA statements.

\subsection{Study Characteristics}

Study characteristics are summarized in Table 1. The total sample was 79. Of these, 41 belonged to the CA group, and 38 were the control group. The average age ranged from 16.7 to 17.5 years old. The sample was comprised exclusively of football players.

Table 1. Characteristics of the sample.

\begin{tabular}{|c|c|c|c|c|}
\hline RCT & Group & Sample Size and Sex & Age* & Condition \\
\hline \multirow{2}{*}{ Kohavi et al., 2018 [21] } & CA & 14 (males) & & \multirow[b]{2}{*}{ Young football athletes } \\
\hline & CG & 12 (males) & 17.5 (1.1) & \\
\hline \multirow{2}{*}{ Harøy et al., 2017 [22] } & CA & 17 (males) & $16.7(0.9)$ & \multirow{2}{*}{ Elite football players } \\
\hline & CG & 16 (males) & $16.9(1.0)$ & \\
\hline \multirow{2}{*}{ Ishøi et al., 2016 [14] } & CA & 10 (males) & 17.3 (SD not reported) & \multirow{2}{*}{ Sub-elite football players } \\
\hline & CG & 10 (males) & 17.4 (SD not reported) & \\
\hline
\end{tabular}

RCT: Randomized Controlled Trial; CA: Copenhagen Adduction; CG: Control group, * Years (SD).

\subsection{Interventions}

The characteristics of the CA intervention and control group are displayed in Table 2. All studies lasted 8 weeks, sessions per week were 2 or 3 , the number of sets and repetitions varied between 1 to 4 and 3 to 15 respectively, the total number of repetitions ranged from 9 up to 90 and the interventions were performed during pre- and in-season. 
Table 2. Characteristics of the interventions.

\begin{tabular}{|c|c|c|c|c|c|c|c|c|}
\hline RCT & CA Group & $\begin{array}{l}\text { Control } \\
\text { Group }\end{array}$ & Duration & $\begin{array}{c}\text { Sessions } \\
\text { Per } \\
\text { Week }\end{array}$ & Sets & Reps & $\begin{array}{c}\text { Reps Per } \\
\text { Week }\end{array}$ & $\begin{array}{l}\text { Season } \\
\text { Period }\end{array}$ \\
\hline $\begin{array}{l}\text { Kohavi et al., } \\
2018\end{array}$ & $\begin{array}{l}\text { CA resistance } \\
\text { training + RFTS }\end{array}$ & $\begin{array}{l}\text { Mobility } \\
\text { exercises + } \\
\text { RFTS }\end{array}$ & 8 weeks & 2 & $2-4$ & $6-10$ & $24-72$ & In-season \\
\hline \multirow{3}{*}{$\begin{array}{l}\text { Harøy et al., } \\
\quad 2017\end{array}$} & \multirow{3}{*}{$\begin{array}{l}\text { FIFA } 11 \text { (with } \\
\text { CA) + RFTS }\end{array}$} & \multirow{3}{*}{$\begin{array}{c}\text { FIFA 11 } \\
\text { (with NH) + } \\
\text { RFTS }\end{array}$} & \multirow{3}{*}{8 weeks } & \multirow{3}{*}{3} & \multirow{3}{*}{1} & $\begin{array}{c}3-5 \\
\text { (Beginner) }\end{array}$ & 9-15 & \multirow{3}{*}{ Pre-season } \\
\hline & & & & & & $\begin{array}{c}\text { 7-10 } \\
\text { (Intermediate) }\end{array}$ & $21-30$ & \\
\hline & & & & & & $\begin{array}{c}12-15 \\
\text { (Advanced) }\end{array}$ & $36-45$ & \\
\hline $\begin{array}{l}\text { Ishøi et al., } \\
2016\end{array}$ & $\begin{array}{l}\text { CA resistance } \\
\text { training + RFTS }\end{array}$ & RFTS & 8 weeks & 2 & $2-3$ & $6-15$ & $24-90$ & In-season \\
\hline
\end{tabular}

\subsection{Outcome Measures}

This systematic review included articles focused on EHAD. All included studies [14,21,22] measured the EHAD using a hand-held dynamometer and according to the protocol and recommendations by Thorborg et al. [23]. The EHAD measure varied from 2.71 to $4.11 \mathrm{Nm} / \mathrm{kg}$. All three articles reported significant between-group or within-group improvement in EHAD after the treatment $[14,21,22]$. In this regard, two studies observed significant between-group improvement $[14,21]$, while the other one found significant within-group improvements [22] and almost significant $(p=0.06)$ between-group changes.

Results revealed a significant overall effect for EHAD in favor to CA compared to CG ( $Z=2.94$; $p=0.003$ ). The overall between-group mean difference was 0.61 , with a $95 \%$ confidence interval from 0.20 to 1.01 (see Figure 2). The heterogeneity level was large $\left(\mathrm{I}^{2}=68 \%\right)$. The quality of the evidence was low according to the GRADE classification.

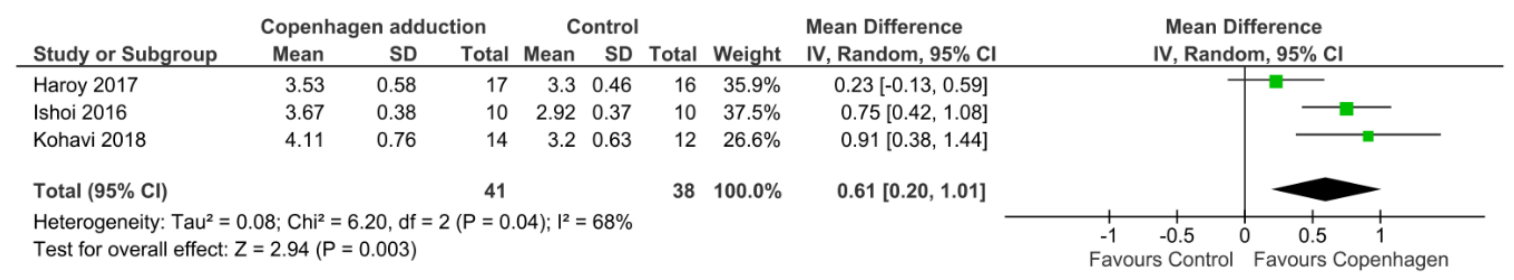

Figure 2. Meta-analysis results of CA exercise intervention on eccentric hip adduction strength (EHAD).

\section{Discussion}

The purpose of this study was to systematically review the scientific literature about the effect of CA exercise training on the EHAD test and to carry out a mini meta-analysis to determine the overall effect as mean difference. Results indicate that eight weeks of training intervention with CA exercise, 2-3 times per week, is effective to improve EHAD in football players [14,21,22]. Therefore, it could be advisable the inclusion of $\mathrm{CA}$ in preventive training programs to potentially reduce injuries in athletes and, specifically, in football players.

The CA is a dynamic high-intensity exercise that includes eccentric contractions and showed high muscle activity of the adductor longus using surface electromyography [12], so it could be recommended in the prevention of adductor and/or groin injuries in athletes. Previous studies observed that additional eccentric exercise reduced the rate of overall, new and recurrent muscle injuries in professional and amateur football players [24]. The CA exercise seems to produce high peak electromyography values for abdominal muscles [12] which may explain why preventing programs based on CA exercise are appropriate against groin problems [25]. 
In the three studies included, the CA exercise was performed in football teams and implemented during the pre-season [22] and in-season [14,21]. A previous study of 12-week eccentric hamstring strengthening program observed a similar magnitude of effect, irrespective of its schedule relative to the football training session. In this regard, they found an increase in strength and surface electromyography, but the adaptations differed according to the timing of the injury prevention program [26]. Therefore, future studies should explore if there is additional benefit when CA is applied in different schedules, not only in football but also in other sports, and establish the optimal volume of sets and repetitions.

As can be seen in Table 2, all training programs lasted eight weeks. This means that more studies are recommended to see if the benefits can be obtained with interventions shorter than eight weeks due to the lack of time at training is one of the barriers identified to carry out evidence-based sport injury prevention programs [27], specifically in football [28]. In this regard, it is important to highlight the short time required to carry out the CA intervention, which is usually shorter than $15 \mathrm{~min}$ [21]. Some strengths of the CA exercise are that it seems to be well-tolerated and safe since none of the three articles reported any important problem as consequence of the intervention. There was high compliance with the training: 91\% [14], 90\% [22], and 85\% [21] what explains the adherence and applicability of the training. It is known that eccentric training intervention for muscle injury prevention depends on good compliance to have success [29]. Another strength is that no special equipment is needed, so it can be performed on the pitch or at any training facility.

Some limitations in the present mini meta-analysis can be mentioned. The first one is related to the search strategy since only articles published in English were included. Another limitation can be that the dominant leg was not reported in the study by Kohavi et al. [21], so the right leg was considered as dominant. However, there was not much difference between the right and left legs. Given the low quality of evidence based on GRADE and the large heterogeneity more studies are needed to have a better understanding of CA exercise to enhance EHAD. Moreover, future studies should compare CA exercise with another alternative training programs for adductor injuries prevention including an injury register and assess the effect of CA depending on the athletes' level, since all participants included in this study were adolescents and soccer players.

\section{Practical Applications}

Based on this mini meta-analysis, some recommendations for the training intervention are highlighted: (a) eight weeks, 2-3 times per week, seems to be effective, (b) weekly volume from 9 to 90 reps is enough to achieve a significant improvement. Nevertheless, further studies with different duration, number of sets, repetitions and in athletes from other sports, not only football, could report additional information to these findings.

\section{Conclusions}

The CA is an easy and useful exercise for improving EHAD in youth football players and, consequently, has a positive effect on one of the factors that influence the injury risk. Thus, the inclusion of CA in prevention programs could be an adequate strategy that could help to reduce the injury risk and consequently suppose a decrease in injury incidence in football.

Author Contributions: Conceptualization, J.P.-G., J.C.A. and J.C.-V.; methodology, J.P.-G., S.V., J.C.-V. and D.C.-M.; software, J.C.A., and D.C.-M.; formal analysis, J.P.-G., S.V., J.C.A., J.C.-V., and D.C.-M.; investigation, J.P.-G., S.V., J.C.A., J.C.-V., M.Á.G.-G. and D.C.-M.; data curation, J.P.-G., J.C.A. and J.C.-V.; writing-original draft preparation, J.P.-G., S.V. and D.C.-M.; writing-review and editing, J.P.-G., S.V., J.C.A., J.C.-V., M.Á.G.-G. and D.C.-M.; supervision, J.P.-G., S.V., J.C.A., J.C.-V., M.Á.G.-G. and D.C.-M. All authors have read and agreed to the published version of the manuscript.

Funding: This research received no external funding.

Conflicts of Interest: The authors declare no conflict of interest. 


\section{References}

1. Rodas, G.; Bove, T.; Caparros, T.; Langohr, K.; Medina, D.; Hamilton, B.; Sugimoto, D.; Casals, M. Ankle Sprain Versus Muscle Strain Injury in Professional Men's Basketball: A 9-Year Prospective Follow-up Study. Orthop. J. Sports Med. 2019, 7, 2325967119849035. [CrossRef] [PubMed]

2. Williams, S.; Trewartha, G.; Kemp, S.; Stokes, K. A meta-analysis of injuries in senior men's professional Rugby Union. Sports Med. 2013, 43, 1043-1055. [CrossRef] [PubMed]

3. Ekstrand, J.; Hagglund, M.; Walden, M. Epidemiology of muscle injuries in professional football (soccer). Am. J. Sports Med. 2011, 39, 1226-1232. [CrossRef] [PubMed]

4. Rogowski, I.; Creveaux, T.; Genevois, C.; Klouche, S.; Rahme, M.; Hardy, P. Upper limb joint muscle/tendon injury and anthropometric adaptations in French competitive tennis players. Eur. J. Sport Sci. 2016, 16, 483-489. [CrossRef]

5. Edouard, P.; Branco, P.; Alonso, J.M. Muscle injury is the principal injury type and hamstring muscle injury is the first injury diagnosis during top-level international athletics championships between 2007 and 2015. Br. J. Sports Med. 2016, 50, 619-630. [CrossRef]

6. Netto, D.C.; Arliani, G.G.; Thiele, E.S.; Cat, M.N.L.; Cohen, M.; Pagura, J.R. Prospective Evaluation of Injuries occurred during the Brazilian Soccer Championship in 2016. Rev. Bras. Ortop. 2019, 54, 329-334. [CrossRef]

7. Nicholas, S.J.; Tyler, T.F. Adductor muscle strains in sport. Sports Med. 2002, 32, 339-344. [CrossRef]

8. Emery, C.A.; Meeuwisse, W.H.; Powell, J.W. Groin and abdominal strain injuries in the National Hockey League. Clin. J. Sport Med. 1999, 9, 151-156. [CrossRef]

9. Tyler, T.F.; Nicholas, S.J.; Campbell, R.J.; McHugh, M.P. The association of hip strength and flexibility with the incidence of adductor muscle strains in professional ice hockey players. Am. J. Sports Med. 2001, 29, 124-128. [CrossRef]

10. Tegner, Y.; Lorentzon, R. Ice hockey injuries: Incidence, nature and causes. Br. J. Sports Med. 1991, 25, 87-89. [CrossRef]

11. Krommes, K.; Bandholm, T.; Jakobsen, M.D.; Andersen, L.L.; Serner, A.; Holmich, P.; Thorborg, K. Dynamic Hip Adduction, Abduction and Abdominal Exercises from the Holmich Groin-Injury Prevention Program Are Intense Enough to Be Considered Strengthening Exercises-a Cross-Sectional Study. Int. J. Sports Phys. Ther. 2017, 12, 371-380. [PubMed]

12. Serner, A.; Jakobsen, M.D.; Andersen, L.L.; Holmich, P.; Sundstrup, E.; Thorborg, K. EMG evaluation of hip adduction exercises for soccer players: Implications for exercise selection in prevention and treatment of groin injuries. Br. J. Sports Med. 2014, 48, 1108-1114. [CrossRef] [PubMed]

13. Hagglund, M.; Walden, M.; Magnusson, H.; Kristenson, K.; Bengtsson, H.; Ekstrand, J. Injuries affect team performance negatively in professional football: An 11-year follow-up of the UEFA Champions League injury study. Br. J. Sports Med. 2013, 47, 738-742. [CrossRef] [PubMed]

14. Ishoi, L.; Sorensen, C.N.; Kaae, N.M.; Jorgensen, L.B.; Holmich, P.; Serner, A. Large eccentric strength increase using the Copenhagen Adduction exercise in football: A randomized controlled trial. Scand. J. Med. Sci. Sports 2016, 26, 1334-1342. [CrossRef]

15. Moher, D.; Shamseer, L.; Clarke, M.; Ghersi, D.; Liberati, A.; Petticrew, M.; Shekelle, P.; Stewart, L.A. Preferred reporting items for systematic review and meta-analysis protocols (PRISMA-P) 2015 statement. Syst. Rev. 2015, 4, 1. [CrossRef]

16. Gopalakrishna, G.; Mustafa, R.A.; Davenport, C.; Scholten, R.J.; Hyde, C.; Brozek, J.; Schünemann, H.J.; Bossuyt, P.M.; Leeflang, M.M.; Langendam, M.W. Applying Grading of Recommendations Assessment, Development and Evaluation (GRADE) to diagnostic tests was challenging but doable. J. Clin. Epidemiol. 2014, 67, 760-768. [CrossRef]

17. Schmidt, F.L.; Oh, I.-S.; Hayes, T.L. Fixed-versus random-effects models in meta-analysis: Model properties and an empirical comparison of differences in results. Br. J. Math. Stat. Psychol. 2009, 62, 97-128. [CrossRef]

18. Hsiao, M.-Y.; Hung, C.-Y.; Chang, K.-V.; Chien, K.-L.; Tu, Y.-K.; Wang, T.-G. Comparative effectiveness of autologous blood-derived products, shock-wave therapy and corticosteroids for treatment of plantar fasciitis: A network meta-analysis. Rheumatology 2015, 54, 1735-1743. [CrossRef]

19. Chang, K.-V.; Wu, W.-T.; Han, D.-S.; Özçakar, L. Ulnar nerve cross-sectional area for the diagnosis of cubital tunnel syndrome: A meta-analysis of ultrasonographic measurements. Arch. Phys. Med. Rehabil. 2018, 99, 743-757. [CrossRef] [PubMed] 
20. Higgins, J.P.; Thompson, S.G. Quantifying heterogeneity in a meta-analysis. Stat. Med. 2002, 21, 1539-1558. [CrossRef]

21. Kohavi, B.; Beato, M.; Laver, L.; Freitas, T.T.; Chung, L.H.; Dello Iacono, A. Effectiveness of Field-Based Resistance Training Protocols on Hip Muscle Strength Among Young Elite Football Players. Clin. J. Sport. Med. 2018. [CrossRef] [PubMed]

22. Haroy, J.; Thorborg, K.; Serner, A.; Bjorkheim, A.; Rolstad, L.E.; Holmich, P.; Bahr, R.; Andersen, T.E. Including the Copenhagen Adduction Exercise in the FIFA 11+ Provides Missing Eccentric Hip Adduction Strength Effect in Male Soccer Players: A Randomized Controlled Trial. Am. J. Sports Med. 2017, 45, 3052-3059. [CrossRef] [PubMed]

23. Thorborg, K.; Couppé, C.; Petersen, J.; Magnusson, S.; Hölmich, P. Eccentric hip adduction and abduction strength in elite soccer players and matched controls: A cross-sectional study. Br. J. Sports Med. 2011, 45, 10-13. [CrossRef] [PubMed]

24. Petersen, J.; Thorborg, K.; Nielsen, M.B.; Budtz-Jorgensen, E.; Holmich, P. Preventive effect of eccentric training on acute hamstring injuries in men's soccer: A cluster-randomized controlled trial. Am. J. Sports. Med. 2011, 39, 2296-2303. [CrossRef]

25. Haroy, J.; Clarsen, B.; Wiger, E.G.; Oyen, M.G.; Serner, A.; Thorborg, K.; Holmich, P.; Andersen, T.E.; Bahr, R. The Adductor Strengthening Programme prevents groin problems among male football players: A cluster-randomised controlled trial. Br. J. Sports Med. 2019, 53, 150-157. [CrossRef]

26. Lovell, R.; Knox, M.; Weston, M.; Siegler, J.C.; Brennan, S.; Marshall, P.W.M. Hamstring injury prevention in soccer: Before or after training? Scand. J. Med. Sci. Sports 2018, 28, 658-666. [CrossRef]

27. Donaldson, A.; Callaghan, A.; Bizzini, M.; Jowett, A.; Keyzer, P.; Nicholson, M. A concept mapping approach to identifying the barriers to implementing an evidence-based sports injury prevention programme. Inj. Prev. 2019, 25, 244-251. [CrossRef]

28. Joy, E.A.; Taylor, J.R.; Novak, M.A.; Chen, M.; Fink, B.P.; Porucznik, C.A. Factors influencing the implementation of anterior cruciate ligament injury prevention strategies by girls soccer coaches. J. Strength Cond. Res. 2013, 27, 2263-2269. [CrossRef]

29. Goode, A.P.; Reiman, M.P.; Harris, L.; DeLisa, L.; Kauffman, A.; Beltramo, D.; Poole, C.; Ledbetter, L.; Taylor, A.B. Eccentric training for prevention of hamstring injuries may depend on intervention compliance: A systematic review and meta-analysis. Br. J. Sports Med. 2015, 49, 349-356. [CrossRef] 